\title{
Frequency and wavelength dependences of electro-optic coefficients in inorganic crystals
}

\author{
M. ABARKAN \\ J.P. SALVESTRINI \\ M.D. FONTANA \\ M. AILLERIE
}

\author{
Laboratoire Matériaux Optiques, Photonique et Systèmes, FRE CNRS 2304, \\ Université de Metz et Supélec, 2, rue E. Belin, 57070 Metz, France
}

\begin{abstract}
The frequency dependence of electro-optic coefficients in congruent and stoichiometric $\mathrm{LiNbO}_{3}, \mathrm{LiTaO}_{3}$, $\beta-\mathrm{BaB}_{2} \mathrm{O}_{4}$ and $\mathrm{KTiOPO}_{4}$ was determined and compared to each other for the first time in a wide frequency range from DC up to $150 \mathrm{MHz}$. The measurements have been investigated as a function of laser wavelength from 0.5 to $1.3 \mu \mathrm{m}$. Clamped and unclamped electro-optic coefficients as well as the acoustic contribution have been established for each crystal.
\end{abstract}

1

\section{Introduction}

It is well known that the dependence on the frequency of the electro-optic (EO) coefficients in crystals reflects the various physical processes contributing to the EO effect according to the working frequency. Thus, organic materials generally do not exhibit any dispersion in frequency, since the EO coefficient measured at low frequency (below the $\mathrm{MHz}$ range) has nearly the same value as that deduced from the nonlinear optical coefficient, so that it is generally established that in organic materials the EO properties have an electronic origin only [1].

The contribution arising from the optical phonons is usually not negligible in dielectric inorganic crystals. This socalled ionic contribution is therefore responsible for dispersion above several GHz. The electro-optic dispersion is still more complicated in piezoelectric crystals and leads in the low-frequency range to an additional contribution of the EO coefficients via the elasto-optical process [2].

The EO coefficients as measured below and above the piezo-resonances are the constant-stress $r^{\mathrm{T}}$ (unclamped) and constant-strain (clamped) $r^{\mathrm{S}}$ coefficients respectively. The knowledge of these two coefficients is useful, for instance, for the determination of the driving voltages corresponding to the switching on or off of the cavity in Pockels cells. The step between $r^{\mathrm{T}}$ and $r^{\mathrm{S}}$, denoted by $r^{\mathrm{a}}$, corresponds to the acoustic contribution. The amplitude of this step is directly linked to the strength of the acoustic resonances and thus can give information about the piezo-electric effect in the material.
The accurate determination of the EO coefficients over a wide frequency range therefore gives information on the physical mechanism at the origin of the EO properties and on the working frequencies or frequency ranges for various applications.

We have undertaken to measure the frequency dependence of EO coefficients for several inorganic piezoelectric crystals within a wide frequency range up to $150 \mathrm{MHz}$. For this, we used a new method based upon the EO response of a crystal to a step voltage [3]. Here we use this method in a one-beam Sénarmont setup to report, for the first time, the frequency dispersion of the EO coefficients in a wide range for crystals well known for their use or interest in EO devices: congruent and stoichiometric $\mathrm{LiNbO}_{3}$ (LNc and LNs respectively), congruent $\mathrm{LiTaO}_{3}$ (LT), $\mathrm{KTiPO}_{4}$ (KTP) and $\beta-\mathrm{BaB}_{2} \mathrm{O}_{4}$ (BBO). The values of the EO coefficients were obviously earlier reported in the literature. However, the large accuracy and the abilities of our measurement technique allow us to provide the values of the EO coefficients with a large confidence, in a wide frequency range and for visible and near-infrared wavelengths.

We derive the acoustic contribution to the EO coefficients from the difference between clamped and unclamped values and we discuss the ability of each crystal for its use in EO devices such as a Pockels cell. In this way, it is thus possible to deduce characteristics of figures of merit needed for various laser applications using these nonlinear single crystals.

\section{$2 \quad$ Experimental setup}

The experimental setup used to measure the EO coefficients was based on the Sénarmont arrangement. The crystal followed by a fixed $\lambda / 4$ plate was located between a linear polarizer and an analyzer. The polarizer was set to yield laser light polarized at 45 degrees to the principal axis of the crystal. The $\lambda / 4$-plate axis was also set at 45 degrees to the crystal axis, while the analyzer was oriented at an azimuthal angle $\beta$. The transfer function of the light intensity transmitted through the setup can be written as [4]:

$T=\frac{1-\gamma \sin (\Gamma-2 \beta)}{2}$ 
where $\beta$ is the angular position of the analyzer and $\Gamma$ is the phase shift introduced by the EO crystal respectively, whereas

$\gamma=\frac{I_{\max }-I_{\min }}{I_{\max }+I_{\min }}$

is the contrast, which is usually considered as a constant parameter. The phase shift $\Gamma_{E}$ and the birefringence $\Delta n(E)$ induced by an electric field $E$ are given by

$\Gamma_{E}=\frac{2 \pi L}{\lambda} \Delta n(E)=\frac{\pi}{\lambda} L n_{\mathrm{eff}}^{3} r_{\mathrm{eff}} E$,

where $L$ is the length of the crystal along the beam-propagation direction, $\lambda$ is the wavelength and $n_{\text {eff }}$ and $r_{\text {eff }}$ are the effective refractive index and EO coefficient respectively.

We used the time-response method (TRM), which is able to provide the frequency dispersion of the EO coefficients. In particular, we determined the clamped (or at constant strain) $r^{\mathrm{S}}$ coefficient and the unclamped (or at constant stress) $r^{\mathrm{T}}$ coefficient. The method consists in the measurement of the time response of the EO crystal to a voltage step. For this, a large voltage (up to $1 \mathrm{kV}$ ) pulse was applied to the crystal with a short rise time (a few nanoseconds) in order to obtain the value of the coefficient $r^{\mathrm{S}}$ and with a long duration (several $\mu \mathrm{s})$ to determine the value of the coefficient $r^{\mathrm{T}}$.

The voltage-induced intensity variation is a maximum at the linear part of the transfer function. At this working point, the time dependence of the variation of the transmitted beam intensity $\Delta i(t)$ induced by the applied voltage $\Delta V(t)$ yields the time response of the EO crystal under test as

$\Delta i(t)=\frac{\pi n_{\mathrm{eff}}^{3} L I_{0}}{2 \lambda d} r_{\mathrm{eff}}(t) \otimes \Delta V(t)$,

where $\otimes$ is the convolution operator, $I_{0}=I_{\max }-I_{\min }$ represents the total intensity shift of the transfer function and $r_{\text {eff }}(t)$ is the value of the EO coefficient. The frequency dispersion of the EO coefficients can be derived from the ratio of the quantities $\Delta i(v)$ and $\Delta V(v)$, which are obtained by the Z-transformation of the signals $\Delta i(t)$ and $\Delta V(t)$ that were measured. We get

$r_{\mathrm{eff}}(\nu)=\frac{2 \lambda d}{\pi n_{\mathrm{eff}}^{3} I_{0} L} \frac{\Delta i(\nu)}{\Delta V(\nu)}$.

We have shown [3] that this technique allows us to obtain the frequency dispersion of the EO coefficient from DC up to at least $150 \mathrm{MHz}$, depending only on the rise time of the voltage pulse.

3

\section{Results}

The method was used to characterize various crystals: a commercial LNc crystal grown by the Czochralski technique, a LNs crystal prepared by the high-temperature topseeded solution-growth technique [5], a BBO crystal grown by the Czochralski technique and commercial LT and KTP crystals. We summarize in Table 1 the different configurations used in our experiments.

To determine the frequency dependence of the EO coefficient, we record both the applied voltage $\Delta V(t)$ and the optical signal $\Delta i(t)$. We derive the frequency variation of the EO parameter $r_{\text {eff }}$ using (4) and (5).

In Fig. 1, we present the EO time response recorded for the different crystals.

The signal exhibits a huge increase immediately after the electric field has been switched on (Fig. 1a). In the longtime range (Fig. 1b) the optical signal oscillates with a period that corresponds to the main piezo-electric frequency resonances. The oscillations tend to disappear for times longer than several tens of $\mu \mathrm{s}$. For any crystal (Fig. 1a) the oscillations do not exist for times shorter than $0.2 \mu$ s since the acoustic waves need more time to propagate across the crystal. The amplitude value of the signal at short times is nearly constant and can be compared to that recorded at long times above several tens of ms. They correspond to the EO coefficients $r^{\mathrm{S}}$ and $r^{\mathrm{T}}$ respectively. The difference between these values yields the acoustic contribution $r^{\mathrm{a}}=r^{\mathrm{T}}-r^{\mathrm{S}}$. The difference should be positive for the configuration $r_{22}$ in LNc, LNs and BBO crystals, but negative for the configurations $r_{22}$ and $r_{\mathrm{c}}$ in a LT crystal. On the contrary, no step was revealed for the configuration $r_{\mathrm{c}}$ in LNc, LNs and KTP crystals.

We present in Fig. 2 the frequency dependence of the EO coefficients deduced from the time response presented in Fig. 1 by using (5).

The frequency range of our measurements in this case is about $150 \mathrm{MHz}$. At higher frequencies the too noisy signal coming from the detection was not considered.

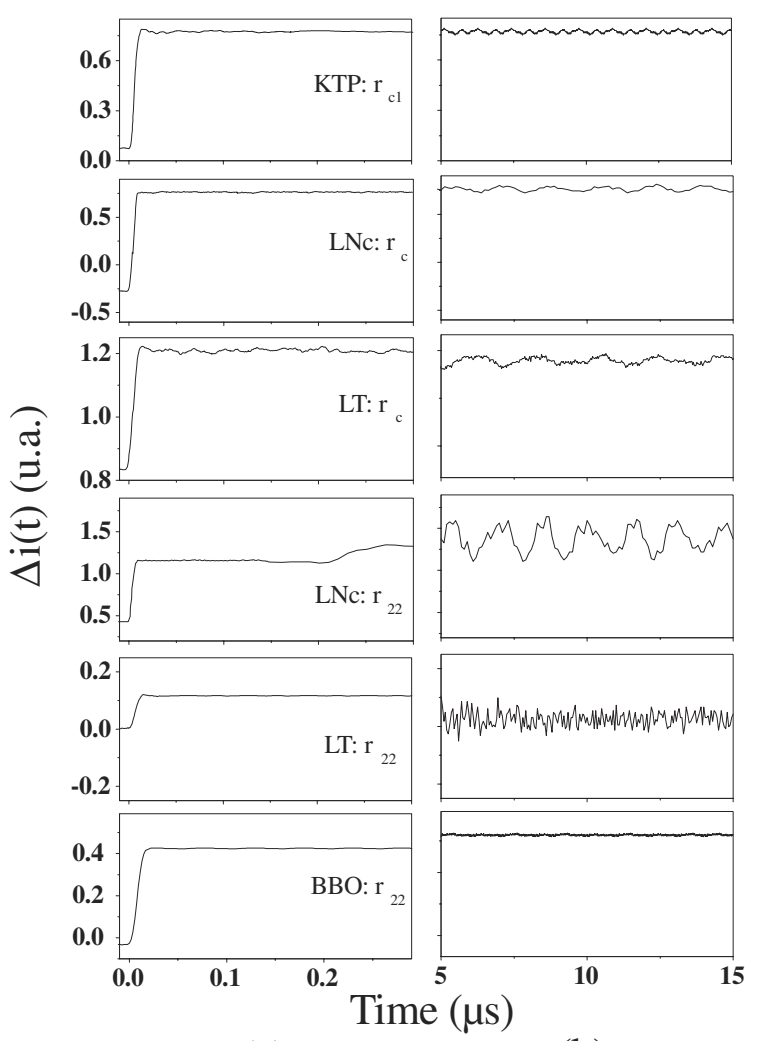

(a)

(b)

FIGURE 1 Time-resolved EO response to a step voltage. a in [0 $\mu \mathrm{s} ; 0.3 \mu \mathrm{s}$ ] time scale and $\mathbf{b}$ in $[5 \mu \mathrm{s} ; 15 \mu \mathrm{s}]$ time scale. Measurements were performed at the wavelength of $632.8 \mathrm{~nm}$ 


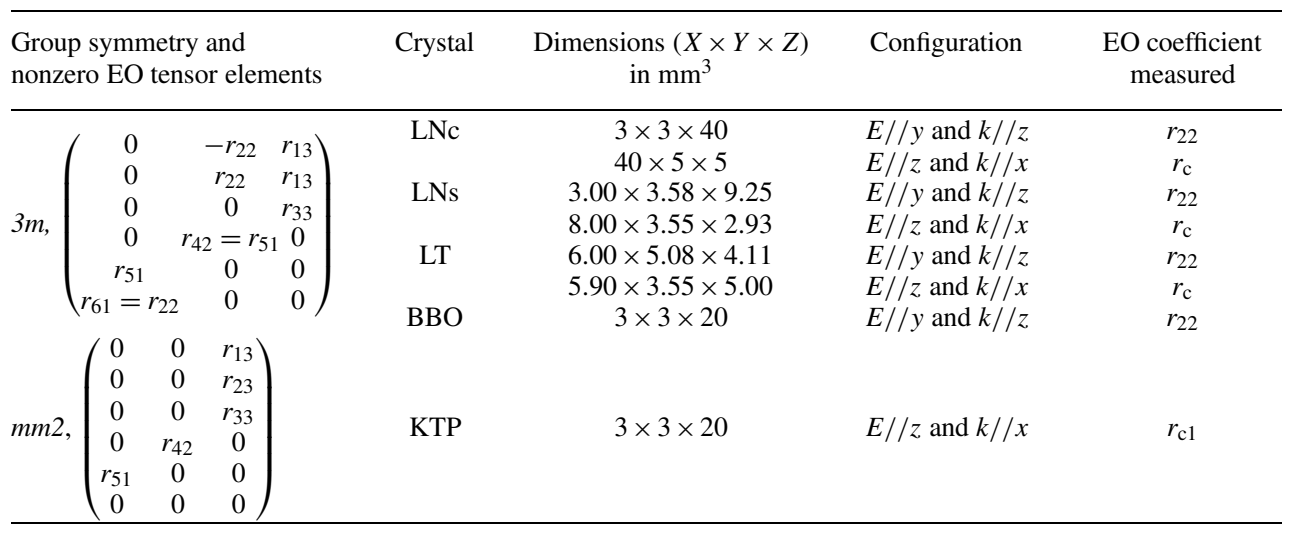

TABLE 1 Characteristics of the EO crystals used in our study. $X, Y$ and $Z$ are the principal axes of the crystals; $k$ and $E$ correspond to the light beam and the electric field directions respectively. Here $r_{\mathrm{c}}=r_{33}-\left(n_{\mathrm{o}}^{3} / n_{\mathrm{e}}^{3}\right) r_{13}$ and $r_{\mathrm{c} 1}=r_{33}-\left(n_{\mathrm{o}}^{3} / n_{\mathrm{e}}^{3}\right) r_{23}$

For each crystal, the main periods of the oscillations generated by the acoustic resonances appearing in the time optical response correspond to the frequencies of the largest peaks existing in the EO frequency dispersion. The values of the unclamped (constant-stress) EO coefficients $r^{\mathrm{T}}$ as deduced from data below the resonances are reported in Table 2 .

The limitation of the pulse duration to a few tens of $\mu \mathrm{s}$ yields a minimum of $10 \mathrm{kHz}$ in the low-frequency range. Since no dispersion is expected at very low frequency, the unclamped value should be equal to the DC value. Above $10 \mathrm{MHz}$ and up to $150 \mathrm{MHz}$ the EO coefficients remain

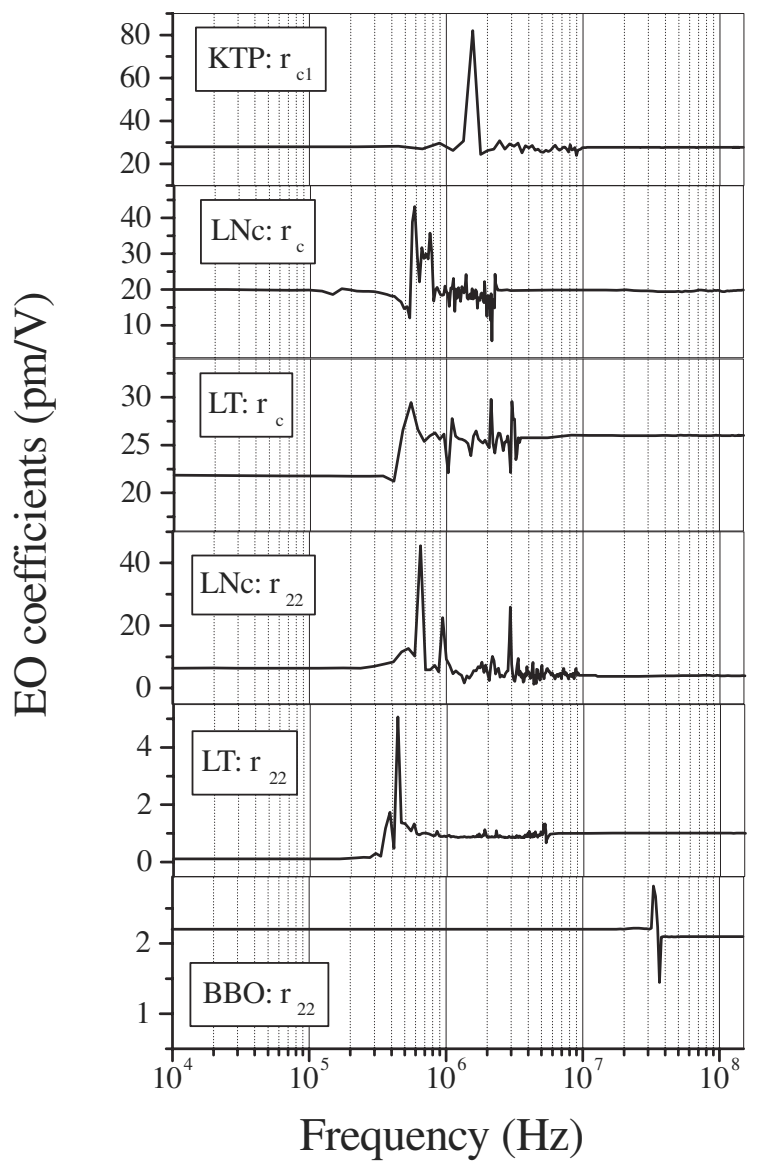

FIGURE 2 Frequency dependence of the EO coefficients at a wavelength of $632.8 \mathrm{~nm}$. These dependences are deduced from the results obtained during the EO time-response measurements constant for each crystal with a value corresponding to the clamped (constant-strain) EO coefficient. These values are in good agreement with those earlier reported [6-9]. Whereas the EO coefficients $r_{22}$ in LNc and LNs and $r_{\mathrm{c}}$ and $r_{22}$ in LT exhibit a large dispersion, the coefficient $r_{\mathrm{c}}$ in KTP, LNc and LNs and the coefficient $r_{22}$ in $\mathrm{BBO}$ are remarkably constant over the whole frequency range.

Additionally, we have derived the acoustic contributions to the EO effect from the difference between unclamped and clamped values $r^{\mathrm{a}}=r^{\mathrm{T}}-r^{\mathrm{S}}$. The acoustic contribution is actually an indirect $\mathrm{EO}$ effect arising from the refractive-index change caused by the crystal deformation in piezo-electric crystals. It can be expressed by the product of the components of the elasto-optic and piezo-electric tensors $p$ and $d$ respectively. The calculation of $r^{\mathrm{a}}$ by this method is rather complicated since it would need the knowledge of the values of all components in the tensors $p$ and $d$. The plot of the frequency dependence of the EO coefficient $r$ constitutes a more direct method to determine $r^{\mathrm{a}}$. Values of $r^{\mathrm{a}}$ obtained from our data are reported in Table 2.

These experimental values of $r^{\mathrm{a}}$ can be checked to be in good agreement, within experimental error, with values calculated from the $p$ and $d$ tensors. In fact, this comparison has been achieved only for the LNc crystal, for which the values

\begin{tabular}{lccc}
\hline Crystal & $\begin{array}{c}\text { Unclamped EO } \\
\text { coefficient } r^{\mathrm{T}} \\
(\mathrm{pm} / \mathrm{V})\end{array}$ & $\begin{array}{c}\text { Clamped EO } \\
\text { coefficient } r^{\mathrm{S}} \\
(\mathrm{pm} / \mathrm{V})\end{array}$ & $\begin{array}{c}\text { Acoustic phonon } \\
\text { contribution } r^{\mathrm{a}} \\
(\mathrm{pm} / \mathrm{V})\end{array}$ \\
\hline LNc & $r_{22}^{\mathrm{T}}=6.4 \pm 0.3$ & $r_{22}^{\mathrm{S}}=3.8 \pm 0.2$ & $r_{22}^{\mathrm{a}}=2.6$ \\
& $r_{\mathrm{c}}^{\mathrm{T}}=20 \pm 1$ & $r_{\mathrm{c}}^{\mathrm{S}}=20 \pm 1$ & $r_{\mathrm{c}}^{\mathrm{a}}=0$ \\
LNs & $r_{22}^{\mathrm{T}}=6.8 \pm 0.4$ & $r_{22}^{\mathrm{S}}=4.5 \pm 0.2$ & $r_{22}^{\mathrm{a}}=2.3$ \\
& $r_{\mathrm{c}}^{\mathrm{T}}=18 \pm 1$ & $r_{\mathrm{c}}^{\mathrm{S}}=18 \pm 1$ & $r_{\mathrm{c}}^{\mathrm{a}}=0$ \\
LT & $r_{22}^{\mathrm{T}}=0.10 \pm 0.01$ & $r_{22}^{\mathrm{S}}=1.0 \pm 0.1$ & $r_{22}^{\mathrm{a}}=-0.90$ \\
& $r_{\mathrm{c}}^{\mathrm{T}}=21.5 \pm 1.5$ & $r_{\mathrm{c}}^{\mathrm{S}}=26.5 \pm 1.5$ & $r_{\mathrm{c}}^{\mathrm{a}}=-5$ \\
KTP & $r_{\mathrm{c} 1}^{\mathrm{T}}=28 \pm 2$ & $r_{\mathrm{c} 1}^{\mathrm{S}}=28 \pm 2$ & $r_{\mathrm{c} 1}^{\mathrm{a}}=0$ \\
BBO & $r_{22}^{\mathrm{T}}=2.2 \pm 0.1$ & $r_{22}^{\mathrm{S}}=2.1 \pm 0.1$ & $r_{22}^{\mathrm{a}}=0.1$ \\
\hline
\end{tabular}

TABLE 2 Unclamped and clamped EO coefficients measured in congruent and stoichiometric $\mathrm{LiNbO}_{3}$, congruent $\mathrm{LiTaO}_{3}, \mathrm{KTP}$ and $\beta$-BBO crystals at a wavelength of $632.8 \mathrm{~nm}$. The contribution $r^{\mathrm{a}}$ is estimated from these measurements 
of $\mathrm{p}$ and $\mathrm{d}$ coefficients were available $\left(r_{22}^{\mathrm{a}}=3.24 \mathrm{pm} / \mathrm{V}\right.$ and $\left.r_{\mathrm{c}}^{\mathrm{a}}=0.04 \mathrm{pm} / \mathrm{V}[10]\right)$.

In addition, the $\lambda$-dispersion of the clamped and unclamped EO coefficients $r_{22}$ in a LNc crystal is reported in Fig. 3 and compared with those in LNs and BBO crystals. Five different wavelengths were used: $0.514 \mu \mathrm{m}$ (Ar laser), $0.633 \mu \mathrm{m}$ (He-Ne laser), $0.815 \mu \mathrm{m}$ (laser diode), $1.064 \mu \mathrm{m}$ and $1.321 \mu \mathrm{m}$ (Nd:YAG laser). It can be observed that both the clamped and unclamped values show a rather strong $\lambda$-dispersion with decreasing wavelength.

The value of the coefficient $r_{22}^{\mathrm{S}}$ is clearly larger in LNs than in LNc in the whole wavelength range. We can assume that this is also the case for the coefficient $r_{22}^{\mathrm{T}}$, although there is an overlap of the error bars.

The clamped and unclamped coefficients $r_{22}$ in LNc and LNs crystals exhibit similar dispersions with wavelength. The values are largely smaller in a BBO crystal for which the coefficients $r_{22}^{\mathrm{T}}$ and $r_{22}^{\mathrm{S}}$ are nearly equal indicating, contrary to $\mathrm{LNc}$ and LNs crystals, weak dependences on frequency and wavelength.

Likewise, we compare in Fig. 4 the $\lambda$-dispersion of the clamped coefficient $r_{\mathrm{c}}^{\mathrm{T}}$ in LNc, LNs, LT and KTP crystals. The dispersion of $r_{\mathrm{c}}^{\mathrm{T}}$ is very similar for all crystals. We note that $r_{\mathrm{c}}^{\mathrm{T}}$ in LNc is larger than in LNs in the whole wavelength range. Our results (at $632.8 \mathrm{~nm}$ ) are in good agreement with data of Méndez et al. [6] and Chah et al. [11] and consistent with values of $r_{\mathrm{c}}^{\mathrm{T}}$ deduced from the measurements of $r_{13}$ and $r_{33}$ [12]. On the contrary, our conclusion is completely opposite to the statement of Fujiwara et al. [13], who reported a value of $r_{\mathrm{c}}^{\mathrm{T}}$ much larger

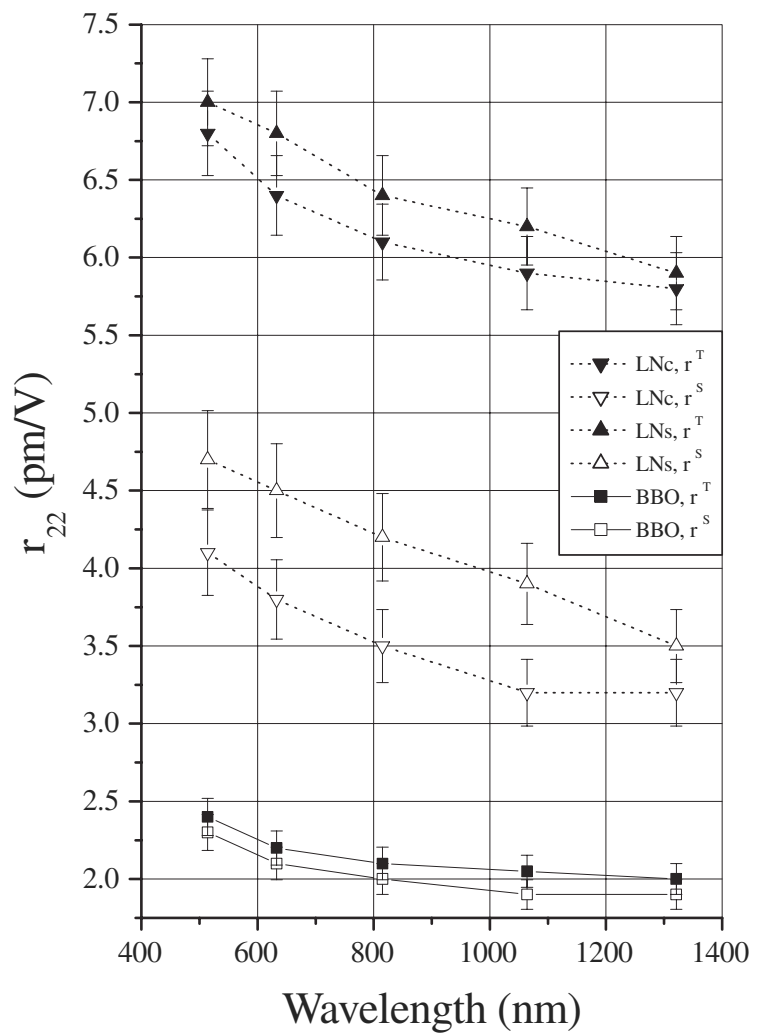

FIGURE 3 Comparison of the wavelength dependence of the EO coefficients $r_{22}^{\mathrm{T}}$ and $r_{22}^{\mathrm{S}}$ in LNc, LNs and BBO crystals

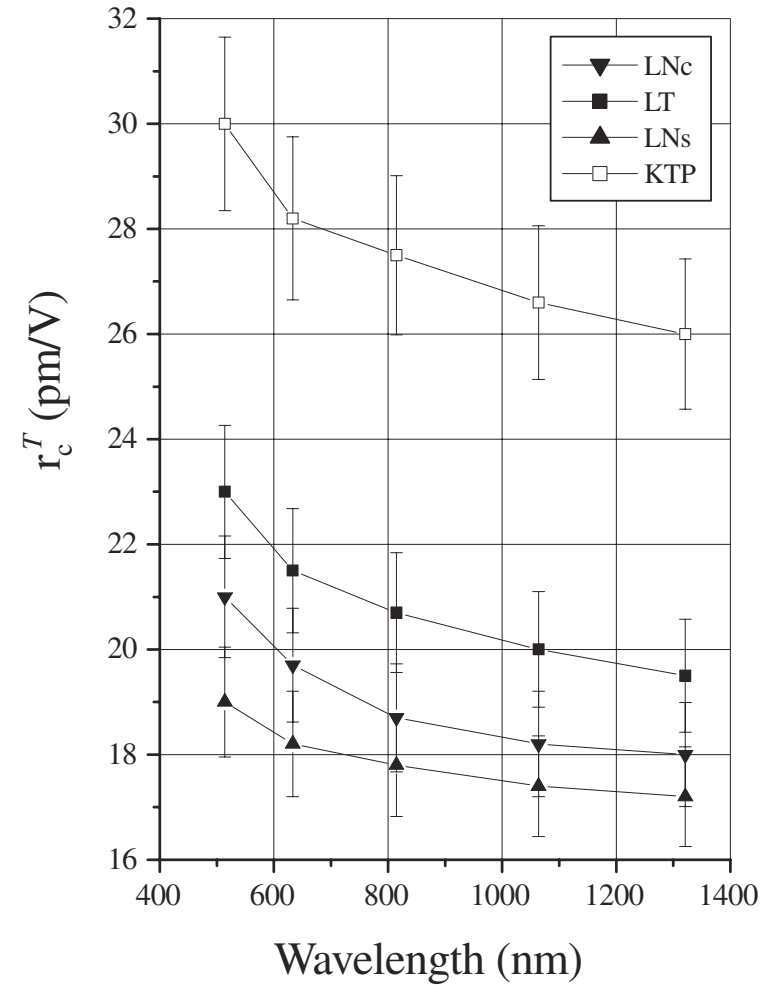

FIGURE 4 Comparison of the wavelength dependence of the EO coefficient $r_{\mathrm{c}}^{\mathrm{T}}$ in LNc, LNs, LT and KTP crystals

in LNs than that in LNc. In addition, a clear discrepancy in the dispersion of $r_{\mathrm{c}}^{\mathrm{T}}$ above $800 \mathrm{~nm}$ is to be noticed between our results and data of Méndez et al., who expected a large decrease of the EO coefficient $r_{\mathrm{c}}^{\mathrm{T}}$ with wavelength.

Data in Table 2 show that the LT crystal, which could be an alternative to $\mathrm{LiNbO}_{3}$ in integrated optics and optoelectronic devices [14], exhibits a very small value of $r_{22}^{\mathrm{T}}$ with a very large acoustic contribution ( $90 \%$ of the total contribution). LT presents as a peculiarity to have clamped coefficients $r^{\mathrm{S}}$ ( $r_{22}$ and $r_{\mathrm{c}}$ as well) larger than the free values $r^{\mathrm{T}}$. The EO coefficient $r_{\mathrm{c}}^{\mathrm{T}}$ in LT possesses a value very close to that in LNc or LNs but much smaller than that in KTP.

\section{4}

\section{Summary}

By means of accurate measurements, we have established the frequency and wavelength dependences of the main electro-optic coefficients of well-known nonlinear optical single crystals: $\mathrm{LiNbO}_{3}$ (in stoichiometric and congruent composition), congruent $\mathrm{LiTaO}_{3}, \mathrm{KTiOPO}_{4}$ and $\mathrm{BBO}$. The knowledge of these characteristics could be of prime importance to define the parameters (half-wave voltage, wavelength, etc.) needed for the use of these materials in devices and applications.

ACKNOWLEDGEMENTS We are grateful to Katalin Polgár for supplying the crystals of lithium niobate and beta barium borate, N. Théofanous for lithium tantalate crystals and D. Lupinski for KTP crystals. This work was sponsored by the Region of Lorraine and the French Ministry of Research. 


\section{REFERENCES}

1 P. Günter: Electrooptic and Photorefractive Materials (Springer, Berlin 1987)

2 J.P. Salvestrini, M.D. Fontana, B. Wyncke, F. Bréhat: Nonlinear Opt. 17, 271 (1997)

3 M. Abarkan, J.P. Salvestrini, M. Aillerie, M.D. Fontana: Appl. Opt. 42, $2346(2003)$

4 L. Guilbert, J.P. Salvestrini, M.D. Fontana, H. Hassan: IEEE J. Quantum Electron. QE-35, 273 (1999)

5 K. Polgár, A. Peter, I. Foldvari: Opt. Mater. 19, 7 (2002)

6 A. Méndez, A. Garcia-Cabanes, E. Diéguez, J.M. Cabrera: Electron. Lett. 35, 498 (1999)
7 E.H. Turner: Appl. Phys. Lett. 8, 303 (1966)

8 C.A. Ebbers: Appl. Phys. Lett. 52, 1948 (1988)

9 P.V. Lenzo, E.H. Turner, E.G. Spencer, A.A. Ballman: Appl. Phys. Lett. 8, 81 (1966)

10 M. Jazbinsek, M. Zgonik: Appl. Phys. B 74, 407 2002)

11 K. Chah, M.D. Fontana, M. Aillerie, P. Bourson, G. Malovichko: Appl. Phys. B 67, 65 (1998)

12 J.A. de Toro, M.D. Serrano, A. Garcia Cabanes, J.M. Cabrera: Opt. Commun. 154, 23 (1998)

13 T. Fujiwara, M. Takahashi, M. Ohama, A.J. Ikushima, Y. Furukawa, K. Kitamura: Electron. Lett. 35, 499 (1999)

14 J.L. Casson, L. Wang, N.J.C. Libatique, R.K. Jain, D.A. Scrymgeour, V. Gopalan, K.T. Gahagan, R.K. Sander, J.M. Robinson: Appl. Opt. 41, 6416 (2002) 